\title{
Lichen planus pigmentosus inversus associated with oral lichen planus
}

\author{
Antonio Carlos Bastos Gomes ${ }^{1}$, César Bimbi', Piotr Brzezinski ${ }^{2,3}$
}

${ }^{1}$ Brazilian Society of Dermatology, Porto Alegre, Brazil, ${ }^{2}$ Department of Physiology and Cosmetology Institute of Biology and Environmental Protection, Pomeranian Academy, Slupsk, Poland, ${ }^{3}$ Department of Dermatology, Provincial Specialist Hospital in Slupsk, Ustka, Poland

Corresponding author: Dr. Antonio Carlos Bastos Gomes, E-mail: bastosdermato@uol.com.br

\begin{abstract}
A 69-year-old lady presented with asymptomatic hyperchromic macules located in the axillary and inguinal folds of six months of evolution. Skin biopsy confirmed the histological pattern of lichen planus leading to diagnosis of Lichen planus pigmentosus inversus (LPPI). She also had white striations of oral lichen planus along the dorsum of the tongue. LPPI is a very rare variant of lichen planus. As far as we know, none of the previously reported patients had coincident lesions on oral cavity. Our case adds one more report of LPPI to the known literature also adding a newness to what was known which is the presence of mucosal lesions in this variant.
\end{abstract}

Key words: Lichen planus; Lichen planus pigmentosus inversus; Axilla; Groin

\section{INTRODUCTION}

Lichen planus pigmentosus (LPP) is an unusual clinical variant of lichen planus (LP). In this form, simetrical coalescent dark-brown macules are distributed in "actinic" pattern over sun exposed areas. Lichen planus pigmentosus inversus (LPPI) display the same hyperpigmented macules but the difference is that it is located on sun protected flexural folds. LPPI is considered an extraordinarily rarer variant with less than 30 cases reported in medical literature so far. Herein we present another case of this rare complaint $[1,2]$.

\section{CASE REPORT}

69 year-old female presenting with asymptomatic diffuse pigmentation in the axillary and inguinal areas of six months of evolution (Figs. 1 and 2). Skin biopsy confirmed the histological pattern of lichen planus [3] leading to diagnosis of Lichen planus pigmentosus inversus (LPPI) (Fig. 3). She also had white striations of oral lichen planus on the dorsum of the tongue (Fig. 4).

\section{DISCUSSION}

Lichen planus displays wide spectrum of clinical variations being 2 of them in the form of large dark macules and patches which are termed pigmentosus. LPP spare scalp, mucosal membranes and nails. The first pigmentosus variant affects face and neck areas of skin types III to VI individuals. The second is Lichen

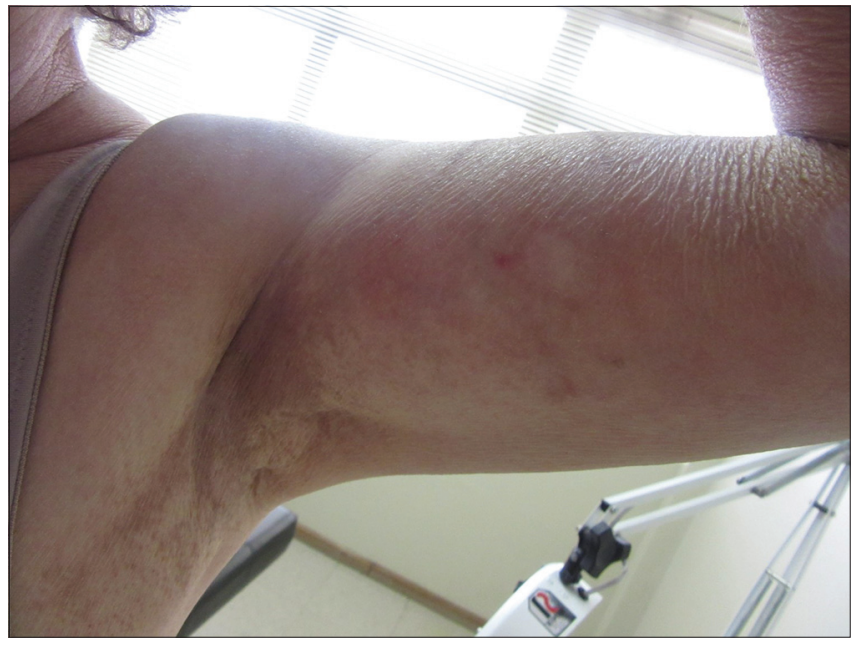

Figure 1: Dark-brown macules in the axillary fold.

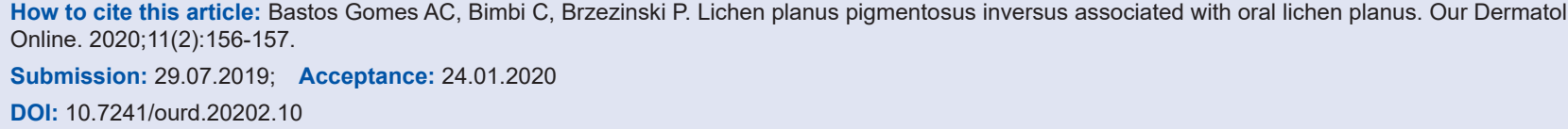




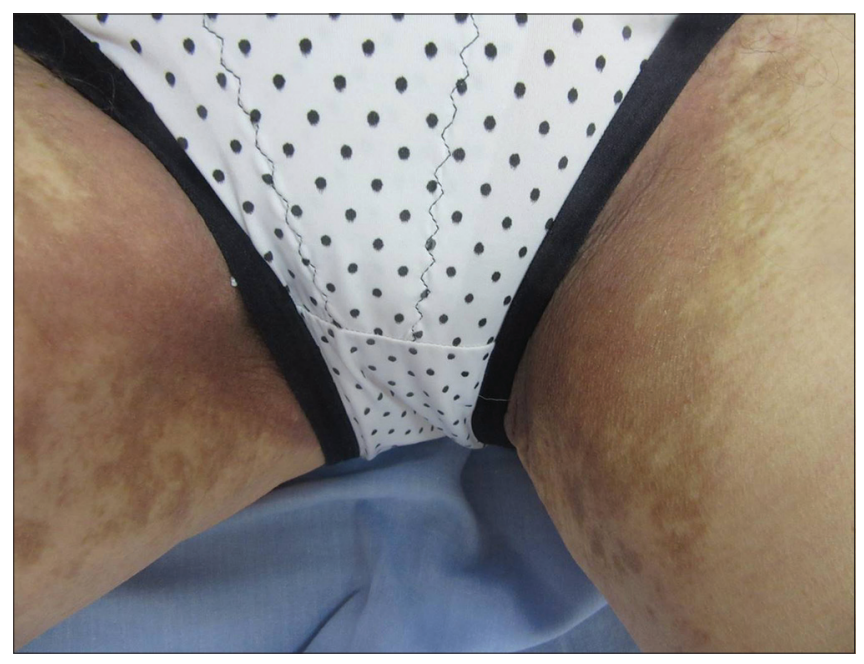

Figure 2: Simetrical coalescent brown patches located on axillary and groin folds.

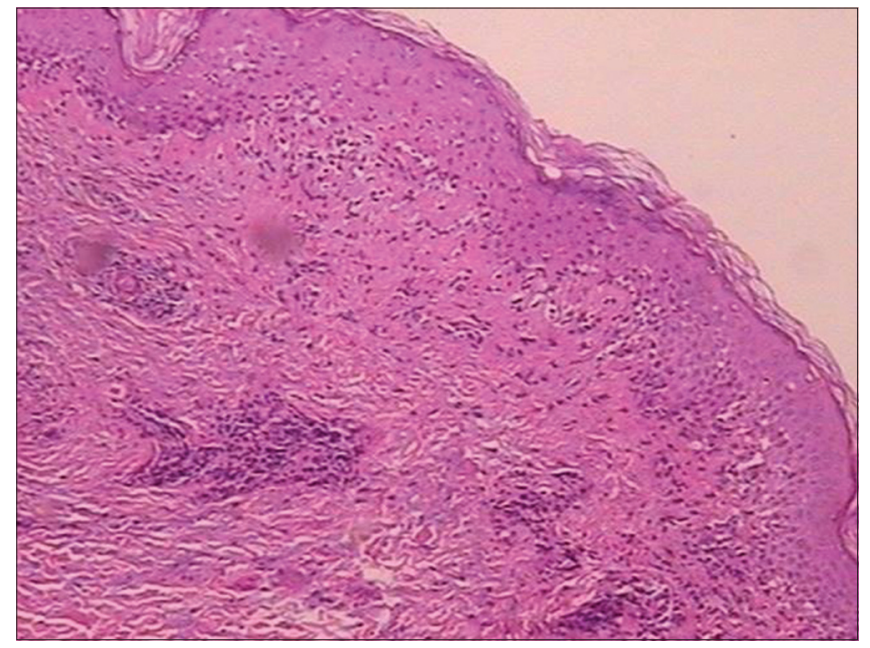

Figure 3: Biopsy of the lesions shows lymphocytic infiltrate lichenoid patterns, with melanophages presence in the papillary dermis.

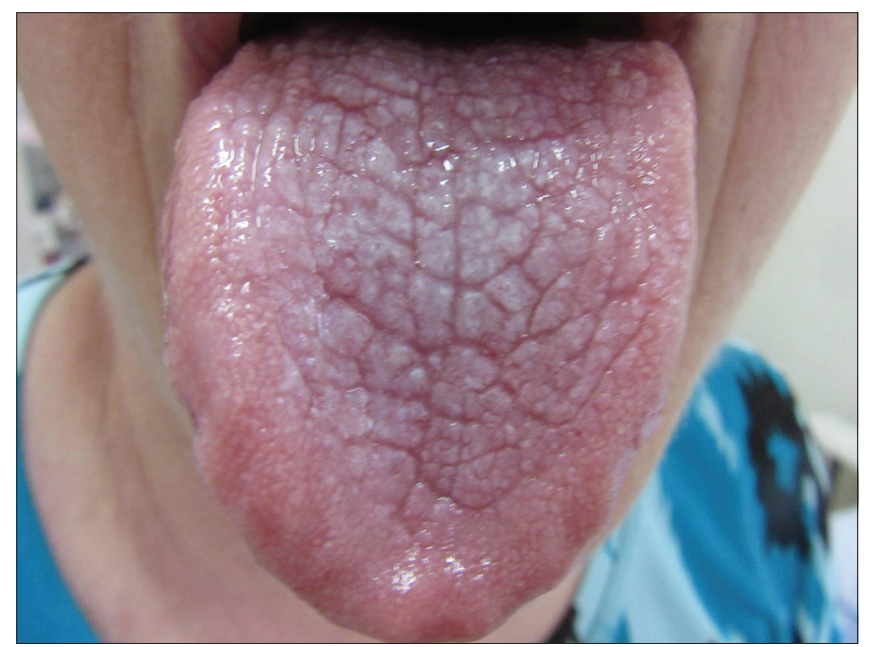

Figure 4: White striations of oral lichen planus along the tongue surface. planus pigmentosus inversus (LPPI), an unusual variant of presentation of LP with less than 30 cases reported in the medical literature [4].

Differently form LPP, Inversus subtype of lichen planus pigmentosus affects sun protected skin-fold areas. In these 2 variations biopsies are equally consistent with liquen planus [5]. The pattern of brown lesions in flexural areas with characteristic lichenoid histology is unique and separates it from other hyperpigmented entities, such as lichen planus actinicus and erythema dyschromicum perstans/ashy dermatosis. In this case hep-C test were negative and she had no records of immunologically mediated disorders or systemic diseases neither family history of pigmentary disorders.

The prognosis of LPPI is benign and the cosmetic appearance is the only concern. By the passage of time the lesions usually tend to stop enlarging and some improvement in the appearance usually happens.

The case presented here differs from all previously reported cases of LPPI for the coexistence of white striations of oral lichen planus lesions in the tongue being the first reported case of LPPI associated with oral lichen planus.

\section{Consent}

The examination of the patient was conducted according to the Declaration of Helsinki principles.

\section{REFERENCES}

1. Gaertner E, Elstein W. Lichen planus pigmentosus-inversus: case report and review of an unusual entity. Dermatol Online J. 2012;18:11.

2. Jung YJ, Lee YH, Lee SY, Lee WS. A case of lichen planus pigmentosus-inversus in a Korean patient. Ann Dermatol. 2011;23:61-3.

3. Orme CM, Kim RH, Brinster N, Elbuluk N, Franks Jr. AG. Lichen planus pigmentosus. Dermatology Online J. 2016;22(12):pii: 13030/ qt3t $24 \mathrm{c} 55 \mathrm{~s}$

4. Ghorbel HH, Badri T, Ben Brahim E, Fenniche S, Benmously R, Mokhtar I. Lichen planus pigmentosus inversus. Indian J Dermatol Venereol Leprol. 2014;80:580.

5. Youssef M, Lahouel I, Korbi M, Soua Y, Marmouch H, Akkari H, et al. Lichen planus pigmentosus and association with autoimmune diseases: A case-control study. Our Dermatol Online. 2019;10:125-30.

Copyright by Antonio Carlos Bastos Gomes, et al. This is an open-access article distributed under the terms of the Creative Commons Attribution License, which permits unrestricted use, distribution, and reproduction in any medium, provided the original author and source are credited. Source of Support: Nil, Conflict of Interest: None declared. 